\title{
High Signal-to-Noise Ratio Mid-Infrared Quasar Spectral Templates
}

\author{
Allison R. Hill ${ }^{1}$, S. C. Gallagher ${ }^{1}$, R. P. Deo ${ }^{1}$, \\ E. Peeters ${ }^{1,2}$ and Gordon T. Richards ${ }^{3,4}$ \\ ${ }^{1}$ The University of Western Ontario, \\ 1151 Richmond Street, London, ON, N6A3K7, Canada \\ email: ahill49@gmail.com \\ ${ }^{2}$ SETI Institute, \\ 189 Bernardo Avenue, Suite 100, Mountain View, CA 94043, USA \\ ${ }^{3}$ Department of Physics, Drexel University, \\ 3141 Chestnut Street, Philadelphia, PA 19104, USA \\ ${ }^{4}$ Max Planck Institut für Astronomie, \\ Königstuhl 17, Heidelberg, Germany 69117
}

\begin{abstract}
Mid-infrared (MIR) quasar spectra exhibit a suite of emission features including high ionization coronal lines from the narrow line region (NLR) illuminated by the ionizing continuum, and hot dust features from grains, as well as polycyclic aromatic hydrocarbons (PAH) features from star formation in the host galaxy. Few features are detected in most spectra because of typically low signal-to-noise ratio $(\mathrm{S} / \mathrm{N})$ data. By generating spectral composites in three different luminosity bins from over 180 Spitzer Ifnfrared Spectrograph (IRS) observations, we boost the $\mathrm{S} / \mathrm{N}$ and reveal important features in the complex spectra. We detect high-ionization, forbidden emission lines in all templates, PAH features in all but the most luminous objects, and broad silicate and graphite features in emission whose strength increases relative to the continuum with luminosity. We find that the intrinsic quasar spectrum for all luminosity templates is consistent, and the differences in the spectra can be explained by host galaxy contamination in the lower luminosity templates. We also posit that star formation may be active in most quasar host galaxies, but the spectral features of star formation are only detectable if the quasar is faint.
\end{abstract}

\section{Introduction}

Quasars are among the most luminous phenomena in the universe, generating substantial radiation across a wide range of the electromagnetic spectrum (from radio to Xrays). The typical quasar spectral energy distribution has two distinct peaks, in the optical/UV and the MIR (see figure 11 in Richards et al. 2006a). The optical/UV peak (the 'big blue bump') is emission from the accretion disk on subparsec scales around the supermassive black hole. The gas temperatures are too high in this region for dust to survive. However, at distances greater than approximately $1 \mathrm{pc}$ from the supermassive black hole (depending on the luminosity of the quasar), temperatures drop such that dust is no longer sublimated. Beyond this point (the dust sublimation radius), grains will be heated by emission from the accretion disk and radiate in the MIR (Antonucci 1993). This thermal emission is the source of the characteristic 'MIR bump' in the quasar SED.

Quasars have been studied extensively in the optical/UV, as relatively unobscured quasars are readily studied from the ground in this regime. As a result, the optical has the advantage of large ( $>100000$ quasar) sample sizes provided by surveys such as the Sloan Digital Sky Survey (SDSS) (Schneider et al. 2010). Only since the advent of spacebased facilities such as Spitzer (Werner et al. 2004) has the sensitivity been available 
to take spectra of similar objects in the MIR. Even with its unprecedented sensitivity, these spectra typically have low signal-to-noise ratios (S/Ns). Using an archival sample of SDSS quasars observed with Spitzer, we constructed spectral composites to boost the $\mathrm{S} / \mathrm{N}$, thus revealing faint features, to characterize typical quasar emission in the MIR.

\section{Quasar Sample Selection and Template Construction}

We coordinate cross-matched the SDSS quasar catalogue (Schneider et al. 2010) with the Spitzer Infrared Spectrograph (IRS; Houck et al. 2004) archive, using a match radius of $2^{\prime \prime}$, yielding 184 low resolution $(R \sim 60-130)$ MIR spectra (see Table 1 of Hill et al. 2013 for a summary of the sample). We selected all data which had short-low and/or long-low modules (although not all objects necessarily had both modules). Although our data span a wide range of luminosities and redshifts, most of our objects occupy the low luminosity, low redshift regime of the parameter space. The details of the data reduction are described in Hill et al. 2013.

The objects were ordered according to their $5.6 \mu \mathrm{m}$ continuum luminosity, and divided equally into three bins of 61 objects per bin. The $\log \left(L_{5.6 \mu m}\right)$ ranges for each tertile are 41.0-43.6, 43.6-44.7, and 44.8-46.1 [erg/s]. For each bin, a template spectrum was generated (Figure 1). Also shown in Figure 1 are the PAHFIT\}model fits for the data. PAHFIT\} is a spectral decomposition code that separates the MIR spectra into its PAH, narrow emission line, and continuum components (Smith et al. 2007). Because PAHfit\}was not designed with AGN in mind, broad gaussian functions were added to fit the broad emission features from silicates at 10 and $18 \mu \mathrm{m}$.

\section{Results and Discussion}

\subsection{Spectral Trends with Luminosity}

A visual inspection of Figure 1 reveals a few qualitative trends with increasing $5.6 \mu \mathrm{m}$ continuum luminosity: the equivalent widths (EWs) of the forbidden lines decreases, the EWs of the PAH features decreases until they are no longer detectable in the most luminous tertile, and the continuum flattens towards longer wavelengths. (For a more quantitative analysis of these trends, and a more in-depth discussion of the narrow lines see Hill et al. 2013.) Because our most luminous objects also tend to be at higher redshifts, we are also able to see an emission bump blueward of $5 \mu \mathrm{m}$ in the most luminous tertile, which was best-fit in PAHFIT with a black body of $750 \mathrm{~K}$. The source of the emission blue-ward of $5 \mu \mathrm{m}$ is from dust with a higher sublimation temperature than silicates, possibly due to graphites located closer to the central engine than the silicate sublimation radius.

\subsection{Quasars and Their Host Galaxies}

The lack of PAH emission in many luminous quasar spectra is often taken to indicate either low levels of star formation in their host galaxies (e.g., Schweitzer et al. 2006) or that the hard ionizing spectrum of the quasar is destroying the requisite molecules (e.g., Genzel et al. 1998). We instead investigate whether the problem could be one of contrast and $\mathrm{S} / \mathrm{N}$, i.e., that the strong MIR continuum of the quasar could be hiding vigorous star formation.

To determine the luminosity required for the strong PAH emission seen in the least luminous template to be detectable in the most luminous quasar template, we took the best-fitting PAH model shown in the bottom panel of Figure 1 and added gaussian noise. To determine the appropriate PAH luminosity, we opted for the most optimistic case: 
the PAH model was assumed to come from the highest luminosity object in the lowest luminosity bin, and the observed template was assigned the lowest luminosity of the objects in the highest luminosity bin. Even in our most optimistic case, there are no prominent $\mathrm{PAH}$ features visible in the residual spectrum made by subtracting the most luminous template model from the template+PAH spectrum. In order for the $11.3 \mu \mathrm{m}$ complex to be detected at $3 \sigma$, the PAH model luminosity had to be scaled up by a factor of 2.5. If we assume that PAH luminosity is correlated with star formation rate (Schweitzer et al. 2006), this suggests there could be a non-negligible amount of star formation activity in quasar host galaxies; the spectral signatures of star formation are just not visible if the quasar is too luminous.

$\mathrm{PAH}$ emission is most prominent in the least luminous tertile, and undetected in the most luminous tertile. This implies that host galaxy contamination is strongest in the least luminous tertile, and the most luminous tertile is more representative of the intrinsic MIR quasar emission. The primary differences between the three templates are therefore consistent with being caused almost entirely by different levels of host galaxy contamination. To illustrate the host galaxy contamination in the least luminous template, we normalized the PAHFIT model continuum from the most luminous template to the $5 \mu \mathrm{m}$ intensity in the least luminous template and subtracted them (Figure 2). Under the assumption that the continuum from the most luminous template is more representative of the intrinsic quasar continuum, the residuals should reflect the host galaxy contribution. We over-plotted a galaxy starburst template from Smith et al. (2007) (their template 3), and normalized the template to the $12.7 \mu \mathrm{m}$ complex of the residuals. The Smith et al. (2007) template does a satisfactory job of reproducing many of the main features of the spectrum as well as its overall shape. Given the diversity of observed star-forming galaxy MIR spectra, we find this level of match to be surprisingly good.

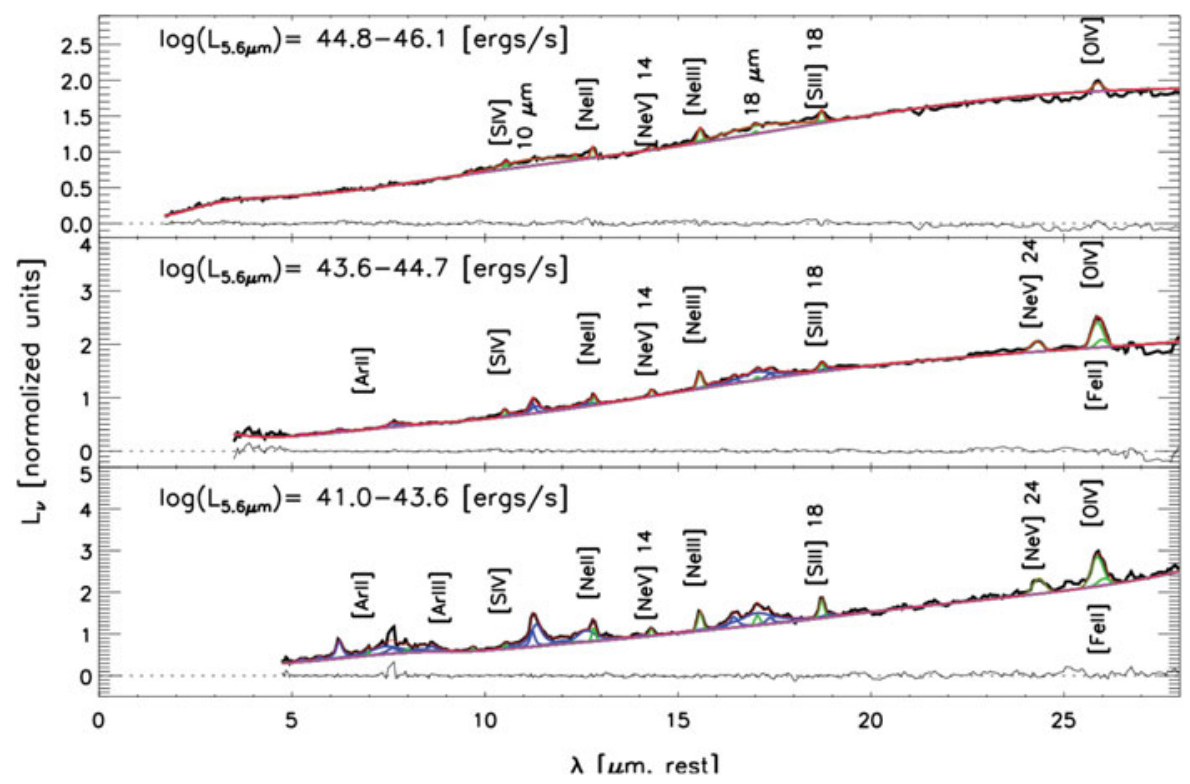

Figure 1. Rest-frame spectral composites with PAHFIT models overplotted, sorted by decreasing luminosity from top to bottom. Model-subtracted residuals straddling the dotted black line at $L_{\nu}=0$. Green and blue curves show the narrow line and PAH features respectively. Magenta is the continuum fit, and red is the total model. Note the absence of PAH features in the top panel; the 10 and $18 \mu \mathrm{m}$ silicate features are only evident in the top panel. 


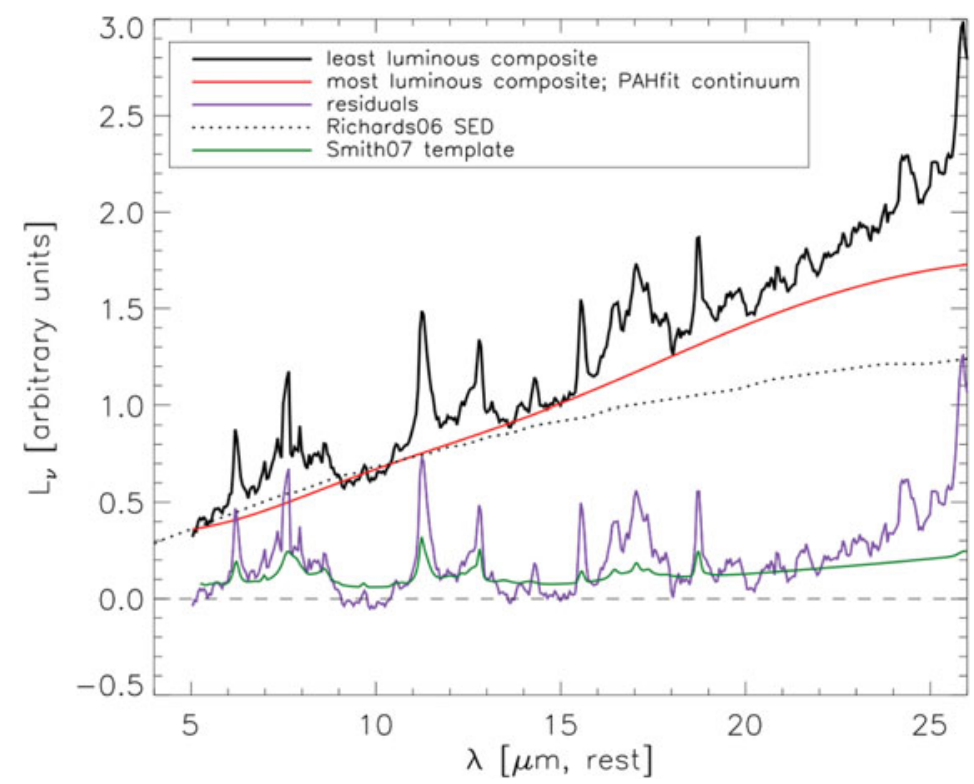

Figure 2. Comparison of the continuum of the most luminous template with the least luminous template. The PAHFIT model continuum of the most luminous template (solid red line) was normalized to the least luminous template at $5 \mu \mathrm{m}$ (solid black line). The Richards et al. (2006a) SED (dotted black line) is overplotted for comparison. The residuals from the subtraction of the PAHFIT model continuum (solid purple line) are above the 0.0 line (dashed black line). A starburst galaxy template from Smith et al. (2007) (solid green curve) is normalized to the $12.7 \mu \mathrm{m}$ complex in the residuals. The features in the residuals can largely be accounted for by star formation in the host galaxy; this suggests that the underlying quasar continua are consistent for all luminosity templates.

Considering the lack of PAH emission, and the results of Figure 2, we suspect that the most luminous template is representative of the true MIR spectrum of a quasar. Furthermore, quasar host galaxies may contain non-negligible levels of star formation, the signatures of which are not detectable if the quasar is too luminous.

\section{References}

Antonucci R. 1993, ApJS 31, 473

Genzel, R., et al. 1998, ApJ 498, 579

Hill, Allison R., Gallagher, S. C., Deo, R. P., Peeters, E., \& Richards, Gordon T. 2013, MNRAS arXiv: 1312.0974

Houck, J. R., et al. 2004, ApJS 154, 18

Richards, G. T., et al. 2006, ApJS 166, 470

Schneider, D. P., et al. 2010, AJ 139, 2360

Schweitzer, M., et al. 2006, ApJ 649, 79

Smith, J. D., et al. 2007, ApJ 656, 770

Werner, M. W., et al. 2004, ApJS 154, 1 